Supporting Information

\title{
Characterization of Nanoparticles in Diverse Mixtures Using Localized Surface Plasmon Resonance and Nanoparticle Tracking by Dark-Field Microscopy with Redox Magnetohydrodynamics Microfluidics
}

Jazlynn C. Sikesł (jwisener@uark.edu), Department of Chemistry and Biochemistry, University of Arkansas, Fayetteville

Kevin Wonner $¥$ (kevin.wonner@rub.de), Faculty of Chemistry and Biochemistry, Ruhr University Bochum, Bochum, Germany 44801

Aaron G. Nicholson (agn001@uark.edu), Department of Chemistry and Biochemistry, University of Arkansas, Fayetteville

Paolo Cignoni (paolo.cignoni@rub.de), Faculty of Chemistry and Biochemistry, Ruhr University Bochum, Bochum, Germany 44801

Ingrid Fritsch* (ifritsch@uark.edu), Department of Chemistry and Biochemistry, University of Arkansas, Fayetteville

Kristina Tschulik* (kristina.tschulik@,rub.de) Faculty of Chemistry and Biochemistry, Ruhr University Bochum, Bochum, Germany 44801

‡: $\quad$ These authors contributed equally.

*: $\quad$ Corresponding authors 
Supporting Information includes discussion of the chemicals and materials, electrode preparation, nanoparticle (NP) motion with and without redox magnetohydrodynamics (RMHD) pumping, NanoTrackJ ${ }^{1}$ settings; table of pumping speeds for individual video sections; table of average nanoparticle diameter, mode diameter of the histograms, and median diameter calculated from 1D MSD of each video segment; table of calculated Ag and $\mathrm{SiO}_{2} @ \mathrm{Au}$ suspension percent ratio; image of the chip used for redox magnetohydrodynamics with dark-field microscopy (RMHDDFM); schematic of the magnet used for RMHD; dark-field microscopy (DFM) image of $\mathrm{SiO}_{2} @ \mathrm{AuNPs}$ in $\mathrm{KNO}_{3}$; tracked NPs in DFM images; applied current waveform and response of potential; plot of NP displacements with and without current in absence of magnetic field; size distribution of AgNPs and $\mathrm{SiO}_{2} @$ auNPs in the absence of a magnetic field and presence of ionic current analyzed by NanoTrackJ, ${ }^{1}$ 1-D mean square displacement (MSD) plots of NP mixture; diffusion coefficient distributions of AgNPs and $\mathrm{SiO}_{2} @$ AuNPs calculated from 1-D MSD; size distributions of $\mathrm{SiO}_{2} @$ AuNPs calculated from 1-D MSD; DFM images obtained during pumping. 


\section{ADDITIONAL EXPERIMENTAL DETAIL}

Chemicals and Materials. UV-treated ultrapure water (Thermo Scientific Barnstead GenPure xCAD Plus) exhibiting a conductivity of $0.055 \mu \mathrm{S} \mathrm{cm}-1(18.2 \mathrm{M} \Omega)$ at $23{ }^{\circ} \mathrm{C}$ was used. The citratecapped silver nanoparticles (AgNP, reported by the manufacturer to be $70 \mathrm{~nm}$ with a zeta potential of $-41 \mathrm{mV})$ and spherical PVP-capped gold-coated silica nanoparticles $\left(\mathrm{SiO}_{2} @ \mathrm{AuNP}\right.$, reported by the manufacturer to be $137 \mathrm{~nm}$ with a zeta potential of $-31 \mathrm{mV}$ ) were purchased from nanoComposix Inc. (Prague, Czech Republic). Potassium nitrate $\left(\mathrm{KNO}_{3}\right)$ of $99.999 \%$ purity and the propylene carbonate (PC) were purchased from Sigma-Aldrich (Germany). The 3,4ethylenedioxythiophene (EDOT) was purchased from Sigma-Aldrich (St. Louis, Missouri, USA). Tetra-n-butylammonium hexafluorophosphate (TBAPF6) of 98\% purity was obtained from Alfa Aesar by Thermo Fisher (Kandel) GmbH. The edge connector (solder contact, 20/40 position, and 0.05 in. pitch) was acquired from Sullins Electronics Corp. (San Marcos, CA, USA). Borosilicate glass wafers (Borofloat 33, $125 \mathrm{~mm} \pm 0.2 \mathrm{~mm}$ outer diameter, $700 \mathrm{um} \pm 100 \mathrm{um}$ thickness) were obtained from Mark Optics, Inc. (Santa Ana, CA, USA). The NdFeB ring magnet was purchased from Conrad Electronic SE. The cover slip (Menzel-Glas) with a dimension of $20 \times 20 \mathrm{~mm}$ was purchased from Thermo Fischer Scientific. Kimwipes (KIMTECH) were obtained from VWR International GmbH (Darmstadt, Germany).

Preparation of poly(3,4-ethylenedioxythiophene) (PEDOT) Modified Electrodes. ${ }^{2}$ Prior to electro polymerization of PEDOT onto the gold band electrodes, ${ }^{3}$ the chip was gently cleaned with a Kimwipe in a sequence of solvents (acetone, ethanol, isopropyl alcohol, and water), rinsed with water, plasma cleaned (Model 1070 NanoClean, Fischione Instruments, Inc.) for 7 min in $25 \% \mathrm{O}_{2}$ and $75 \%$ Ar at $2 \times 10^{-2}$ torr, $46 \mathrm{~W}$ with $1.31 \mathrm{~W}$ reflected, and then immediately immersed into the electropolymerization solution of $0.01 \mathrm{M}$ EDOT and $0.10 \mathrm{M}$ TBAPF $_{6}$ in propylene 
carbonate (PC). When ready for deposition, the chip was removed from the solution, and the contact pads were quickly wiped with a Kimwipe soaked with PC and then water, and wiped dry, without affecting the rest of the solution-wetted chip. The contact pads were inserted into the edge connector and chip was re-immersed sufficiently for the solution to cover the band electrodes. A thick film of PEDOT was then sequentially electropolymerized onto each band electrode, based on a previously described procedure using cyclic voltammetry (CV) with a PalmSens4 galvanostat/potentiostat (Palmsens, Houten, Netherlands), and using a $\mathrm{Ag} / \mathrm{AgCl}$ (3 M KCl) reference electrode and a platinum counter electrode. There were 24 sequential CV cycles performed at $0.050 \mathrm{~V} / \mathrm{s}$ between $-0.4 \mathrm{~V}$ and $1.6 \mathrm{~V}$ and back vs. $\mathrm{Ag} / \mathrm{AgCl}(3 \mathrm{M} \mathrm{KCl})$. The deep purple PEDOT films were further stabilized in a solution of $0.10 \mathrm{M} \mathrm{TBAPF}_{6}$ in $\mathrm{PC}$ by shorting them together and cycling ten times from $0 \mathrm{~V}$ to $0.5 \mathrm{~V}$ and back vs. $\mathrm{Ag} / \mathrm{AgCl}(3 \mathrm{M} \mathrm{KCl})$ at $0.010 \mathrm{~V} / \mathrm{s}$, yielding the typical shape with charging current usually associated with a capacitor. ${ }^{37}$ 


\section{DETAILED DISCUSSION OF DIRECTION AND SPEED OF NANOPARTICLE MOTION WITH AND WITHOUT RMHD PUMPING}

The small ratio of 0.15 for the height of the chamber to the separation between the active band electrodes is expected to produce a relatively parallel ionic current distribution between the electrodes and across the gap (in the y-direction). The MHD force that the ions experience in the presence of the magnetic field (in the z-direction), which is primarily orthogonal to the ionic current, moves them along the length of the gap, between the electrodes (in the x-direction). A momentum transfer occurs to the surrounding fluid around those ions, resulting in "pumping" of fluid in that direction. Because PEDOT holds a finite amount of charge, its pumping duration is

also finite. ${ }^{4}$ However, it can be recharged by reversing the current, which in the presence of a fixed magnetic field, reverses the flow. We took advantage of this behavior in our studies.

There is a slight skew in the direction of the NPs during RMHD pumping off the true $\mathrm{x}$ axis, however. This is most likely attributed to a slight rotation of the RMHD apparatus in the x-y plane relative to the viewing window of the microscope. Another possible reason for this is that a longer PEDOT-covered portion of one of the electrodes could slightly modify the direction of the ionic current across the gap and therefore skew the fluid flow off the central axis. Migration of the negatively-charged NPs toward the positive electrode along the y-axis (in the upward or downward direction of the video frame), superimposed on the RMHD flow along the x-axis, could also produce the skewed flow. However, there was no evidence when the current was applied in the absence of a magnet that such upward/downward motion in the electric field alone was present. The data in Figure S6 substantiate this statement.

One can observe from Video S2 that in the absence of a magnetic field that there exists a global movement of NPs to the left regardless of the application and direction of current, but it is 
much slower than the speed of the RMHD flow attained in the presence of the magnet $\left(<1 \mu \mathrm{m} \cdot \mathrm{s}^{-}\right.$ ${ }^{1}$ ). We do not have a confirmed explanation for this "drift" at this time. This kind of behavior is not unusual in other microfluidic systems, where a small leak or evaporation at the edges of the gasket could draw fluid sideways. The materials contained in the NPs are not paramagnetic, and therefore we do not expect any motion toward higher magnetic field gradients and flux densities, which would be upward (in the z-direction) toward the magnet and sideways toward the edges of the hole. A $1 \mu \mathrm{m} \cdot \mathrm{s}^{-1}$ drift in one $\mathrm{x}$ direction could add to the RMHD flow in that direction and subtract from the flow after RMHD switches direction, yielding a difference of as much as $2 \mu \mathrm{m} \cdot \mathrm{s}^{-}$ ${ }^{1}$. This drift could account for the differences between the forward and reverse RMHD fluid speeds reported in Table S1. 


\section{VIDEOS}

Video S1: Mixture of AgNPs and $\mathrm{SiO}_{2} @ A u N P s$ in $50 \mathrm{mM} \mathrm{KNO} 3$ recorded in the presence of the magnet with an exposure time of $100 \mathrm{~ms}$ and a magnification of $10 \mathrm{x}$. A $50 \mu \mathrm{A}$ current was applied at $\sim 0.5 \mathrm{~s}$. The current was then switched to $-50 \mu \mathrm{A}$ at $\sim 6.5 \mathrm{~s}$ and back to $50 \mu \mathrm{A}$ at $\sim 12.5 \mathrm{~s}$. At $\sim 18 \mathrm{~s}$, the system returned to open circuit potential. The video was originally recorded at 9.9 frames per second, and the playback rate is at 25 frames per second.

Video S2: Mixture of AgNPs and $\mathrm{SiO}_{2} @ A u N P s$ in $50 \mathrm{mM} \mathrm{KNO}$ recorded in the absence of the magnet with an exposure time of $100 \mathrm{~ms}$ and a magnification of $10 \mathrm{x}$. A $50 \mu \mathrm{A}$ current was applied at $\sim 0.5 \mathrm{~s}$. The current was then switched to $-50 \mu \mathrm{A}$ at $\sim 6.5 \mathrm{~s}$ and back to $50 \mu \mathrm{A}$ at $\sim 12.5 \mathrm{~s}$. At $\sim 18 \mathrm{~s}$, the system returned to open circuit potential. The video was originally recorded at 9.9 frames per second, and the playback rate is at 25 frames per second. 


\section{TABLES}

Table S1: RMHD pumping speeds determined from a mixture of AgNPs and $\mathrm{SiO}_{2} @ \mathrm{AuNPs}$ in $50 \mathrm{mM} \mathrm{KNO}_{3}$. The distance traveled for each NP between the first and last frame in the track was assumed to be the distance contributed by the RMHD flow. The speed was calculated by dividing this distance by the amount of time in which the NP track was visible. The averages were calculated by an arithmetic mean.

\begin{tabular}{|c|c|}
\hline Video & Pumping Speed $\left(\mu \mathrm{m} \cdot \mathrm{s}^{-1}\right)$ \\
\hline Forward 1 & Blue/Green \\
\hline Reverse & $13.5 \pm 1.41$ \\
\hline Forward 2 & $14.7 \pm 1.38$ \\
\hline Average & $13.5 \pm 1.65$ \\
\hline & $13.9 \pm 1.48$ \\
\hline Forward 1 & Red/Orange \\
\hline Reverse & $13.3 \pm 1.52$ \\
\hline Forward 2 & $14.5 \pm 1.22$ \\
\hline Average & $13.3 \pm 1.52$ \\
\hline
\end{tabular}


Table S2: Settings used in NanoTrackJ ${ }^{1}$ for the sizing of AgNPs and $\mathrm{SiO}_{2} @ \mathrm{AuNPs}$ in the mixture in $50 \mathrm{mM} \mathrm{KNO}_{3}$.

\begin{tabular}{|c|c|}
\hline Center estimation & Maxima \\
\hline Diffusion-Coefficient Estimator & Covariance \\
\hline Search radius & 15 pixels \\
\hline Min. exp. Nanoparticle diameter & $20 \mathrm{~nm}$ \\
\hline Min. number of steps per track & 15 \\
\hline Temp & $23{ }^{\circ} \mathrm{C}$ \\
\hline Viscosity & $0.9358 \cdot 10^{-4} \mathrm{~Pa} \cdot \mathrm{s}^{*}$ \\
\hline Pixel size & $653 \mathrm{~nm}$ \\
\hline Correct linear drift & True \\
\hline Walker's method & True \\
\hline Tolerance & 30 \\
\hline
\end{tabular}

${ }^{*}$ The values were automatically provided by NanoTrackJ. ${ }^{1}$ 
Table S3: Sizes of the NPs determined from a mixture of AgNPs and $\mathrm{SiO}_{2} @ \mathrm{AuNPs}$ in $50 \mathrm{mM}$ $\mathrm{KNO}_{3}$ in the presence and absence of RMHD flow. The averages were calculated by an arithmetic mean.

\begin{tabular}{|c|c|c|}
\hline Video & Nanoparticle size (nm) & $\begin{array}{c}\text { Number of } \\
\text { nanoparticles } \\
\text { analyzed }\end{array}$ \\
\hline \multicolumn{3}{|c|}{ Blue/Green } \\
\hline Forward 1 & Average 221; Peak max 107.5; Median 167 & 233 \\
\hline Reverse & Average 182; Peak max 72.5; Median 135 & 254 \\
\hline Forward 2 & Average 216; Peak max 77.5; Median 148 & 282 \\
\hline MHD Pumped & Average Peak max 85.8 \pm 19 & - \\
\hline Static & Average 232; Peak max 82.5; Median 150 & 385 \\
\hline \multicolumn{3}{|c|}{ Red/Orange } \\
\hline Forward 1 & Average 247; Peak max 152.5; Median 204 & 174 \\
\hline Reverse & Average 257; Peak max 92.5; Median 169 & 185 \\
\hline Forward 2 & Average 229; Peak max 140; Median 188 & 150 \\
\hline MHD Pumped & Average Peak max 128.3 132 & - \\
\hline Static & Average 174; Peak max 137.5; Median 160 & 254 \\
\hline
\end{tabular}

Each NP was first identified by its localized surface plasmon resonance (LSPR) color and its diffusion coefficient determined by the MSD analysis of its motion during RMHD in forward 1, reverse, and forward 2 passes (in the presence of the magnet) and in the absence of the magnet, but with current applied. The NP sizes were calculated from the diffusion coefficients and a literature value of $8.9 \cdot 10^{-4} \mathrm{~Pa} \cdot \mathrm{s}$ for the viscosity of water at $25^{\circ} \mathrm{C}$ was used. ${ }^{5}$ 


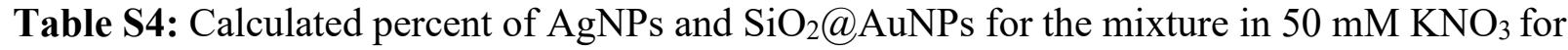
each video segment listed in Table S2. The averages were calculated by an arithmetic mean.

\begin{tabular}{|c|c|c|}
\hline Video & Percent $\mathrm{Ag}$ & Percent $\mathrm{SiO}_{2} @ \mathrm{Au}$ \\
\hline Forward 1 & 56 & 44 \\
\hline Reverse & 58 & 42 \\
\hline Forward 2 & 66 & 34 \\
\hline Static & 54 & 46 \\
\hline Average & $58 \pm 5 \%$ & $42 \pm 5 \%$ \\
\hline
\end{tabular}




\section{FIGURES}

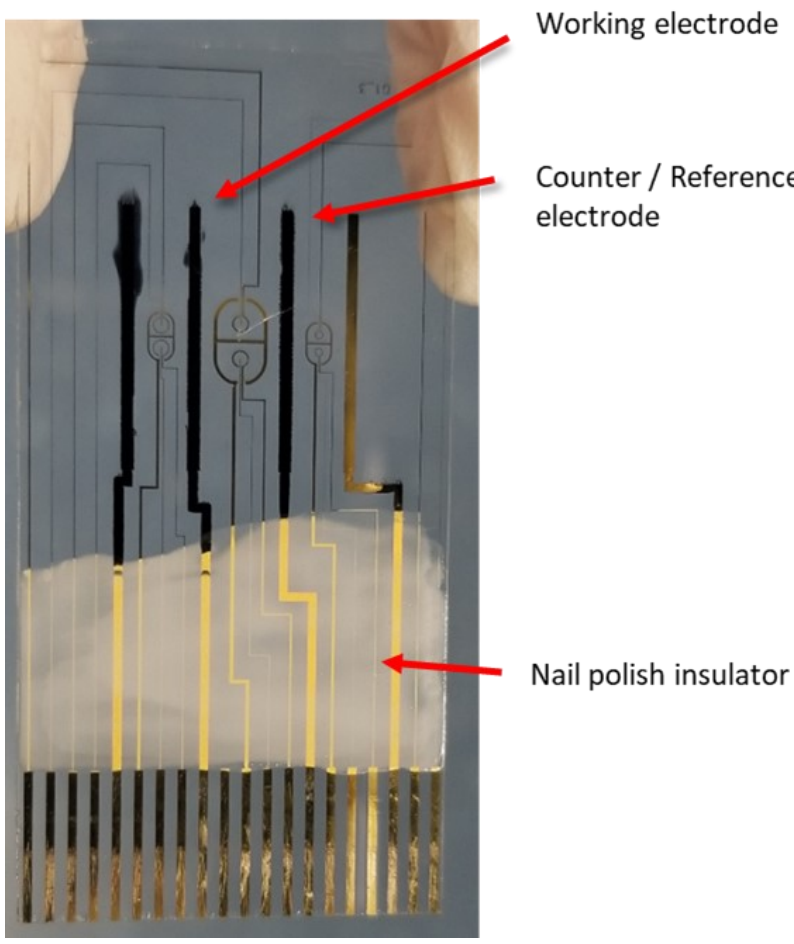

Figure S 1: The $2.45 \mathrm{~cm}$ x $5.08 \mathrm{~cm}$ (1 in x 2 in) borosilicate chip patterned with Au electrodes, leads and contact pads. The two innermost band electrodes used for RMHD-DFM are nominally $650 \mu \mathrm{m}$ wide and spaced $0.47 \mathrm{~cm}$ apart. The original length of the band electrodes, which was defined by an insulating benzocylcobutene (BCB) layer over the leads, was $1.5 \mathrm{~cm}$. However, loss of the insulator yielded a longer portion being part of the active electrode. Thus, the PEDOT, which appears black in the photo, polymerized onto the electrodes and part of the leads, based on the extent of immersion of the electrodes in the EDOT deposition solution. Clear nail polish was used to insulate the gold leads after the deposition of PEDOT. 


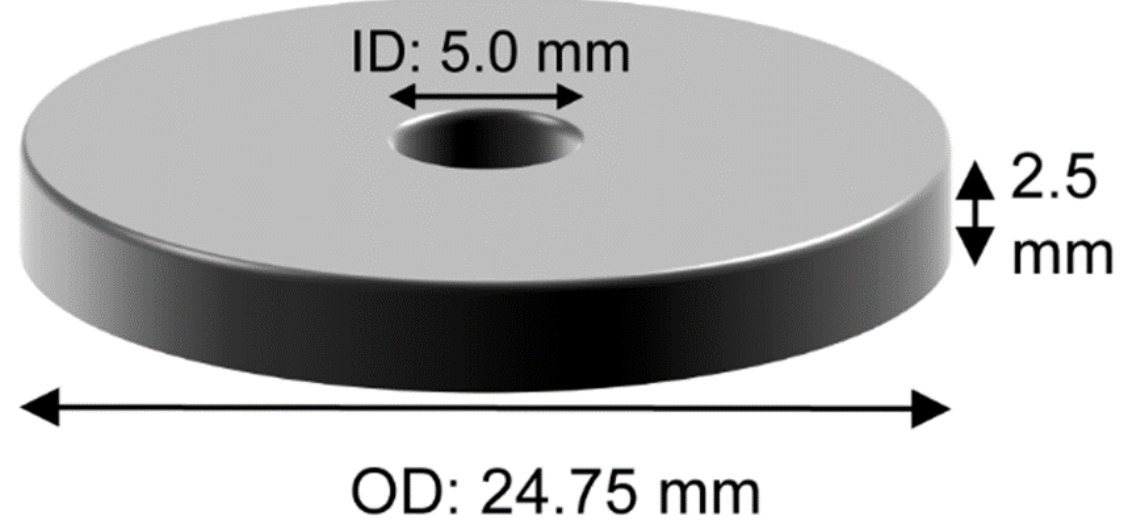

Figure S 2: Dimensions of the ring magnet used for all RMHD-pumped experiments. The lens of the microscope objective was partially inside the $5.0 \mathrm{~mm}$ opening. The magnetic flux measured $270 \mathrm{mT}$. 


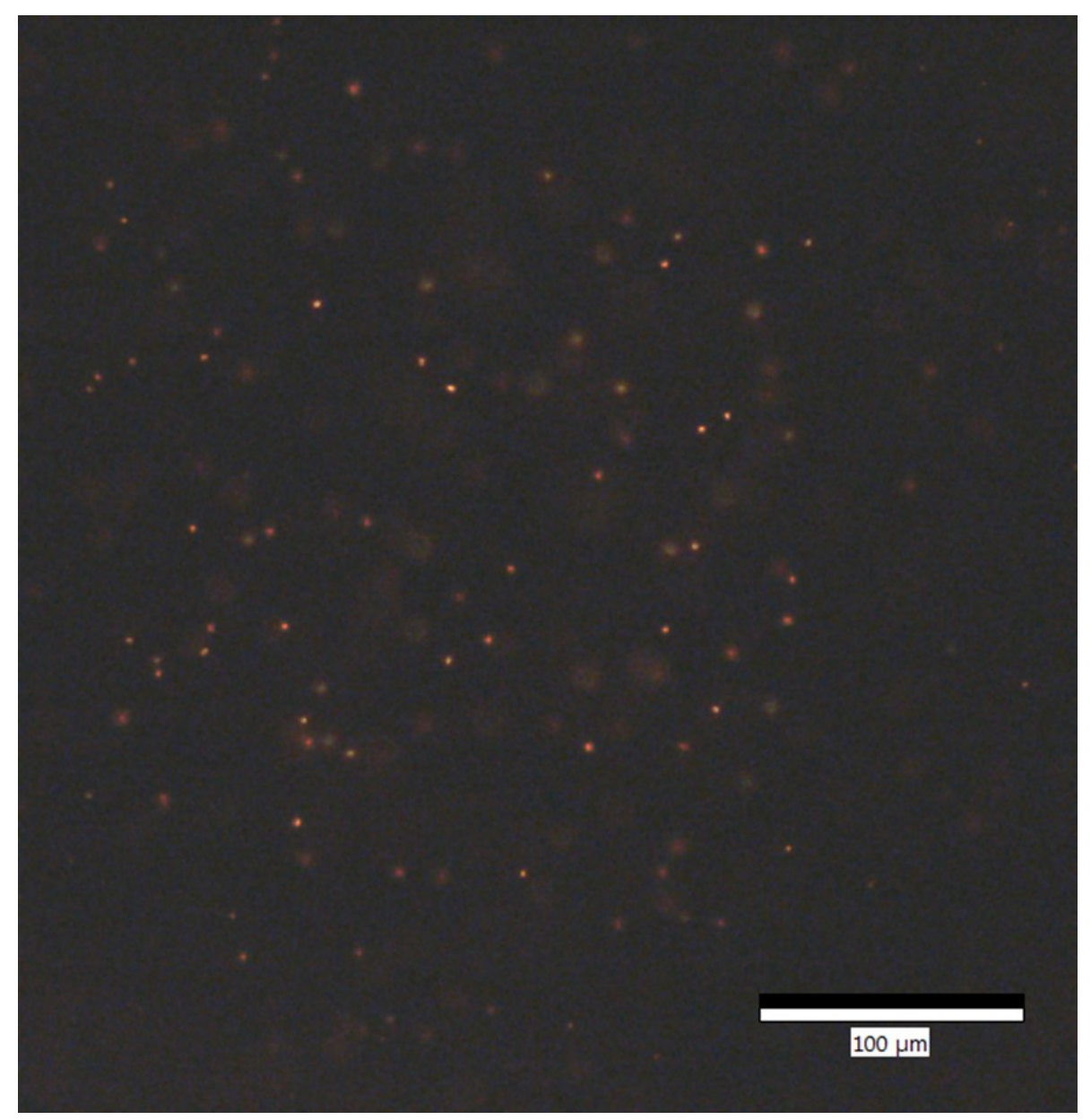

Figure S 3: DFM image depicting pure $140 \pm 9.9 \mathrm{~nm} \mathrm{SiO}{ }_{2} @ A u N P s$ in $50 \mathrm{mM} \mathrm{KNO}$. This frame is from the video taken in the absence of magnetic and electric fields. Hues for the $\mathrm{SiO}_{2} @$ AuNPs were determined from this portion of the experiment. 

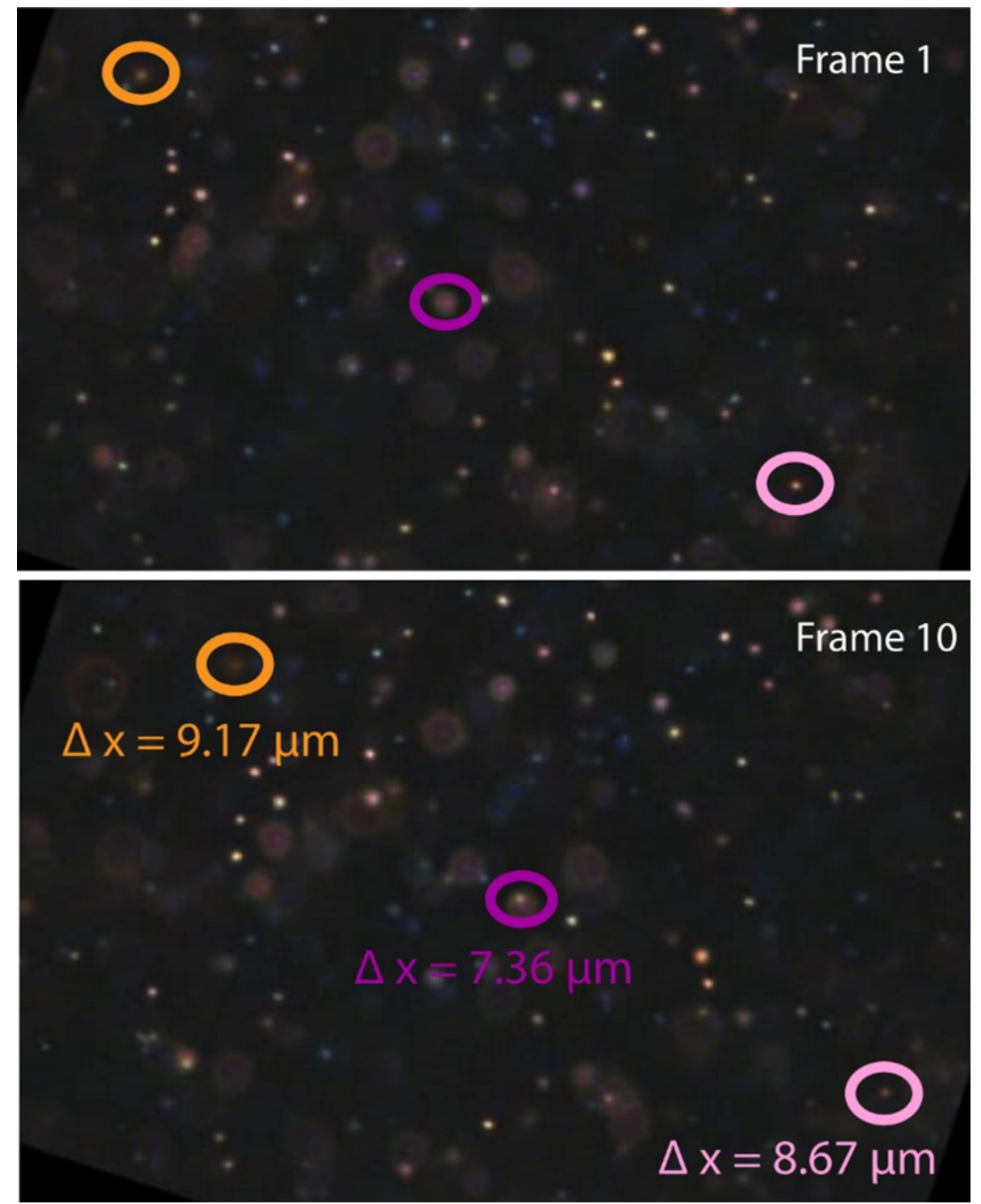

Figure S 4: DFM images showing NPs displacement during RMHD pumping in a NP mixture of AgNPs and $\mathrm{SiO}_{2} @ \mathrm{AuNPs}$ in $50 \mathrm{mM} \mathrm{KNO}_{3}$. The $\Delta \mathrm{x}$ represents the change in the position of each NP between frame 1 (upper photo) and frame 10 (lower photo) of the video recording taken from the forward 1 pumping direction (when $+50 \mu \mathrm{A}$ was applied) at a frame rate of 9.9 fps. The time between these two frames is $1.01 \mathrm{~s}$. 


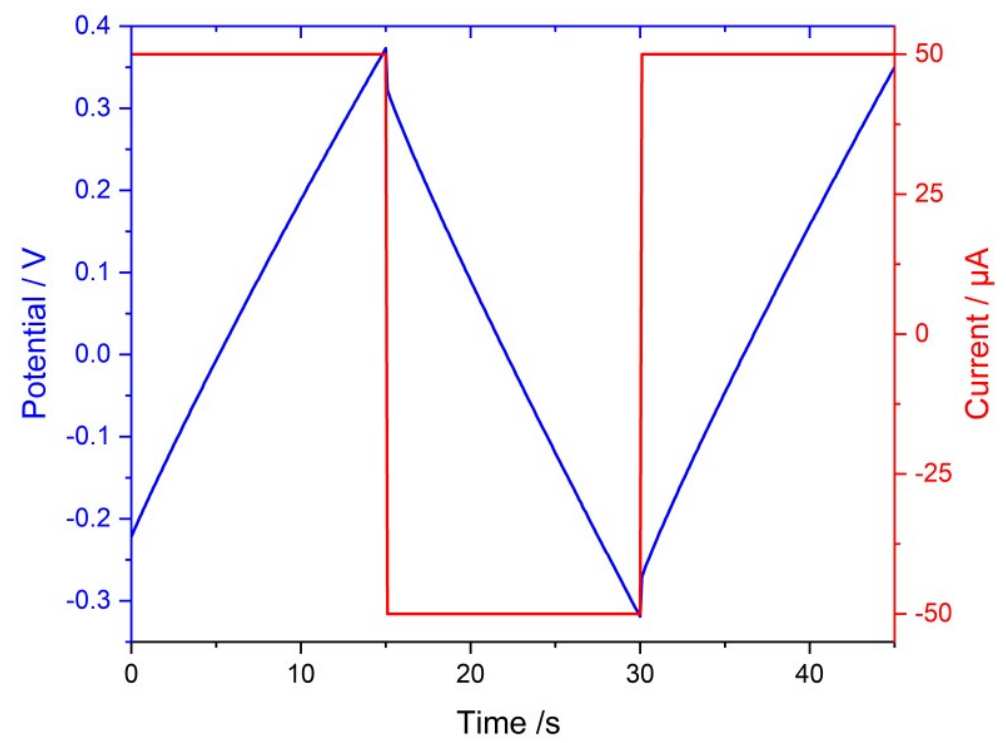

Figure S 5: Multi-chronopotentiometry (blue) response to the applied current waveform (red) between PEDOT-modified electrodes in the RMHD chamber containing $50 \mathrm{mM} \mathrm{KNO}_{3}$ obtained during acquisition of Video 1. (The potential is the measured difference between the two active PEDOT electrodes.) The potential response for the RMHD cell with the PEDOT-modified electrodes behaves like a simple RC circuit as observed previously. ${ }^{4}$ The voltage drop of $0.055 \mathrm{~V}$ at the beginning of each current step represents a cell resistance of $0.055 \mathrm{~V} /\left(5 \times 10^{-5} \mathrm{~A}\right)=11000 \Omega$; the capacitance of the two PEDOT electrodes in series is $\left(5 \times 10^{-}\right.$ $\left.{ }^{5} \mathrm{~A}\right)(15 \mathrm{~s}) /(0.64 \mathrm{~V})=1.2 \mathrm{mF}$ (about $2.4 \mathrm{mF}$ each, if one assumes they are equal in area). 


\section{Electrode}

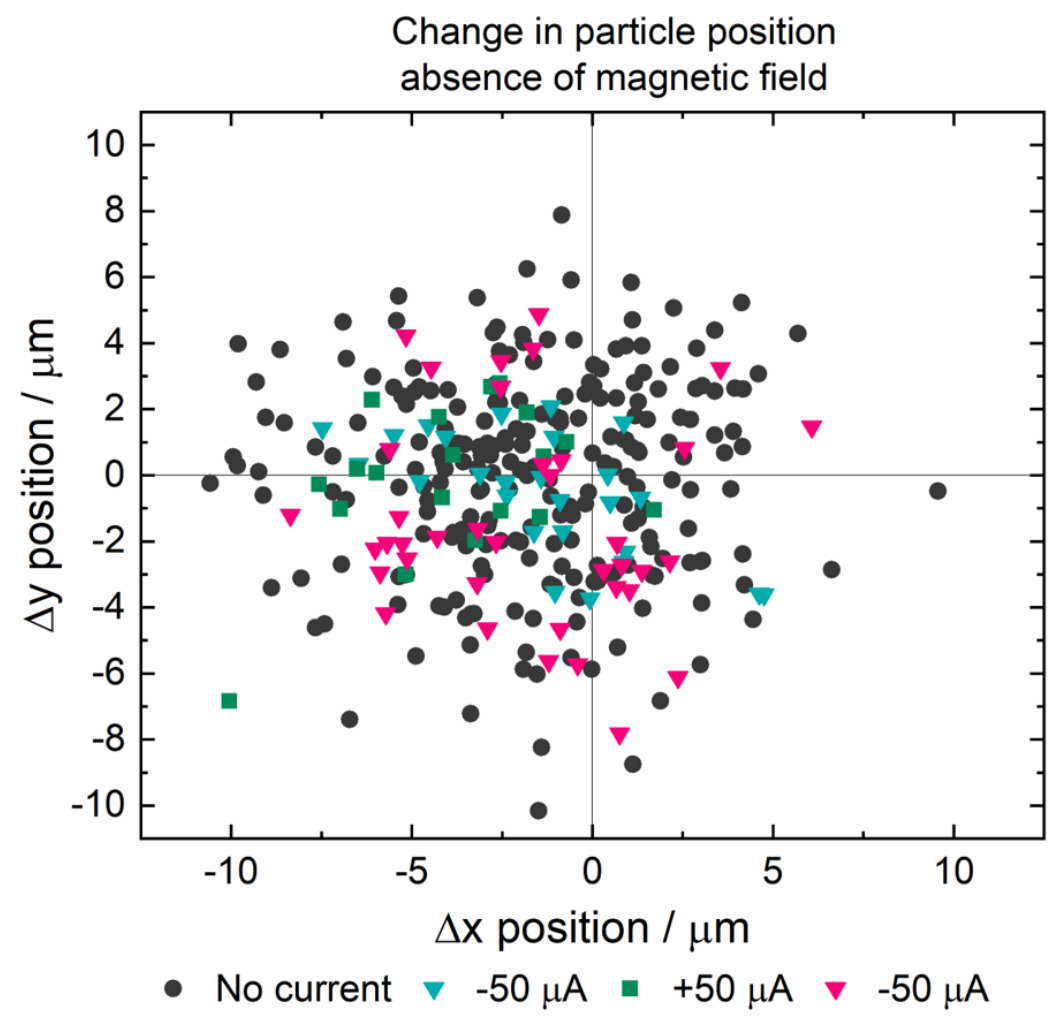

\section{Electrode}

Figure S 6: Net NP movement over $7 \mathrm{~s}$ in the presence and absence of an applied current, but without a magnetic (i.e. no RMHD pumping). The DFM viewing window is centered between the two PEDOT-modified electrodes as schematically depicted in Figure 1 (b) of the main document. The displacement obtained for each NP is represented by a marker at its final position, $(\Delta \mathrm{x}, \Delta \mathrm{y})$, from the origin $(0,0)$. (The electrode widths, gap between the electrodes, and dimensions in the plot are not drawn to-scale.) Displacements are shown for multiple NPs under each of four conditions: no current (black circles), $-50 \mu \mathrm{A}$ (inverted turquoise triangles), $+50 \mu \mathrm{A}$ (green squares), and $-50 \mu \mathrm{A}$ (inverted magenta triangles). There is no net movement toward one electrode or the other when current is applied, indicating that electrophoretic migration of the NPs is not significant. A net negative $\Delta \mathrm{x}$ is present for all conditions, that suggests that a net solution drift is present, but is along the same axis as the RMHD flow when a magnet is added. (This drift will add to the NP speed in one RMHD flow direction and will subtract from the NP speed in the other RMHD flow direction.) 


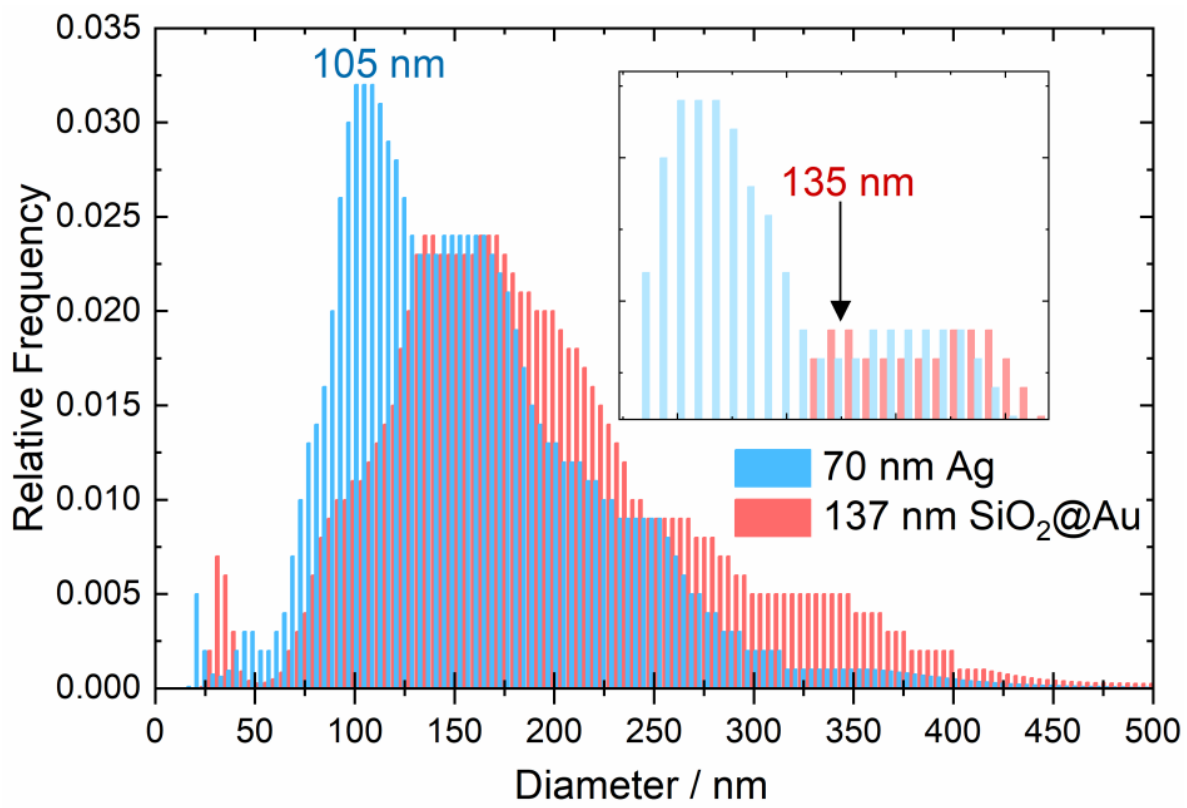

Figure S 7: The size distributions of the AgNPs (blue) and $\mathrm{SiO}_{2} @$ AuNPs (red) in the mixture in 50 mM KNO 3 in the absence of a magnetic field and presence of an ionic current (non-pumped), analyzed in NanoTrackJ. ${ }^{1}$ For clarity, the inset indicates the maximum peak locations for the $\mathrm{SiO}_{2} @$ AuNPs size distribution. 
(a)

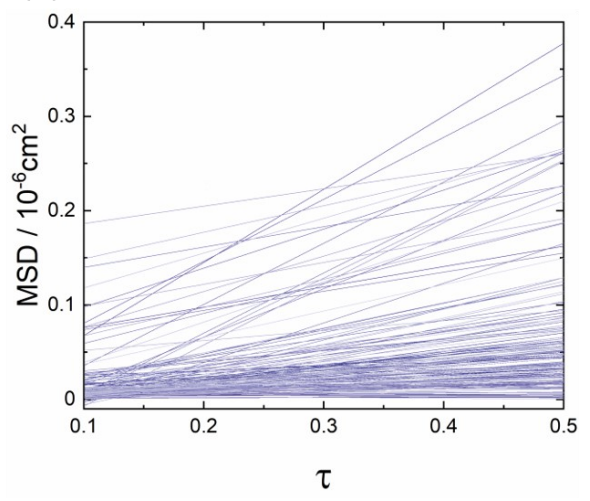

(b)

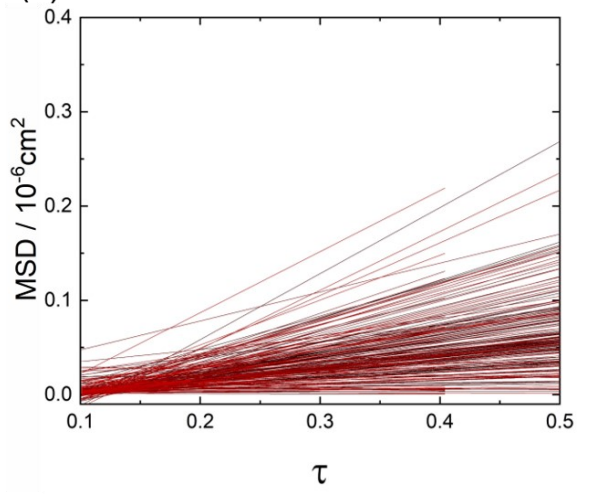

Figure S 8: 1-D MSD plots generated by time steps 1-5 of each NP in the mixture. Plots were generated from the video recording of RMHD pumping in the forward 1 direction of (a) AgNPs and (b) $\mathrm{SiO}_{2} @$ AuNPs. There is one least squares best fit line drawn here per tracked NP and each line was based on five points, each separated from the next by $0.101 \mathrm{~s}$. (Markers are not shown for each point.) Of the NPs tracked for Ag, 210 tracks produced an $\mathrm{R}^{2} \geq 0.9$, and 106 gave an $\mathrm{R}^{2} \geq 0.985$. The blue-colored LSPR was not as intense as the red, making tracking more difficult. Of the NPs tracked for $\mathrm{SiO}_{2} @ \mathrm{Au}, 186$ tracks produced an $\mathrm{R}^{2} \geq 0.9$, and 114 gave an $\mathrm{R}^{2} \geq 0.985$. Lines with negative slopes from some of the NPs were due to tracking errors and removed. 
(a)

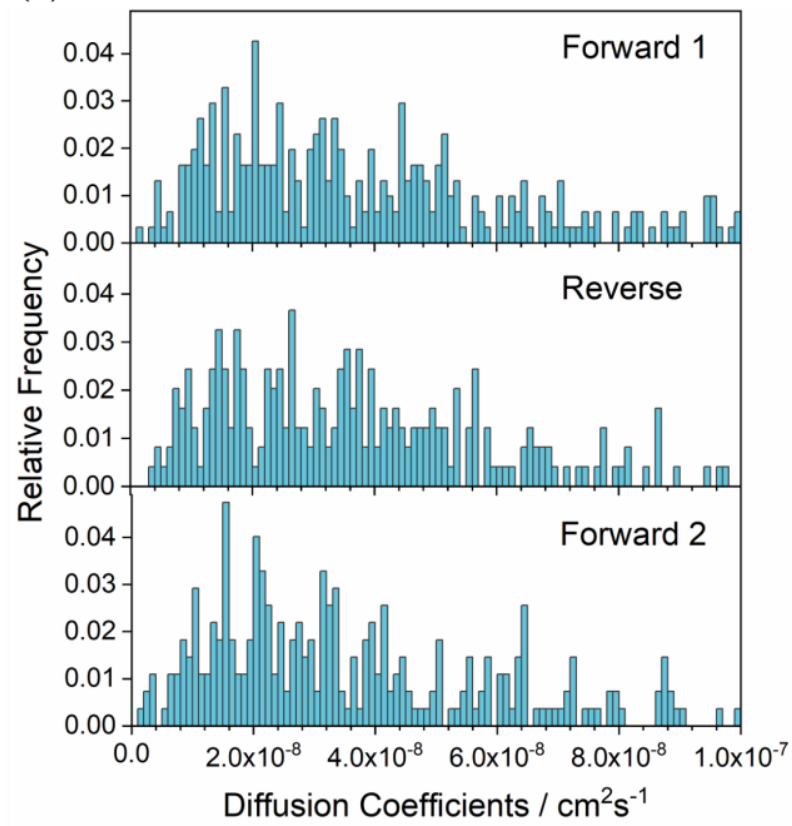

(b)

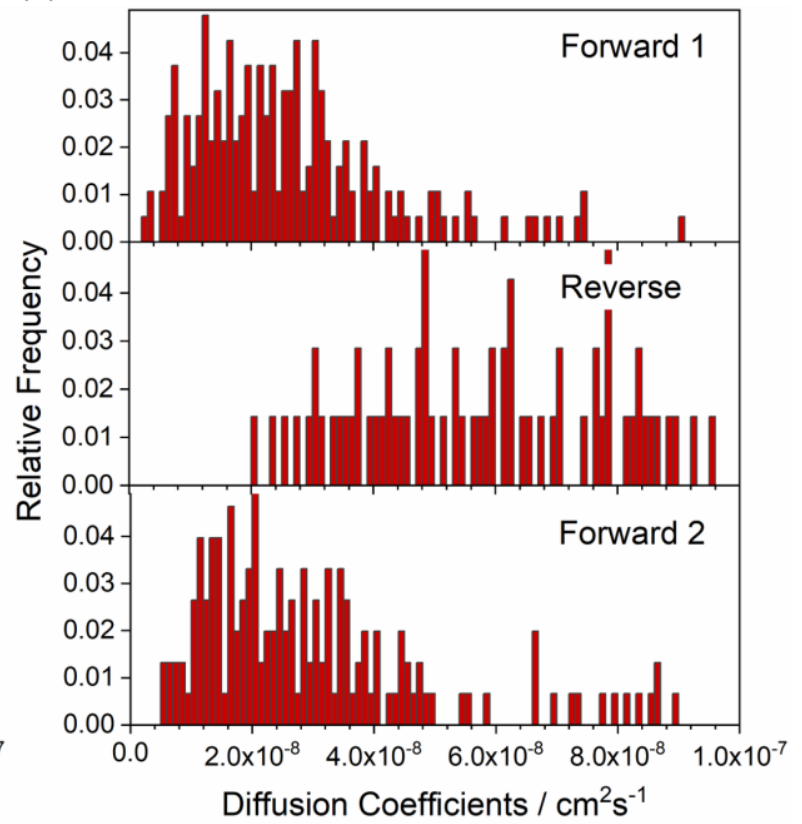

Figure S 9: Size distributions of the NPs with LSPR having (a) hues 56-255, assigned to AgNPs, and (b) hues 0-55, assigned to $\mathrm{SiO}_{2} @ A u N P s$, in the mixture and calculated from the 1-D mean square displacement of the RMHD-pumped (forward 1, reverse, and forward 2) and of the non-pumped (static) suspension 


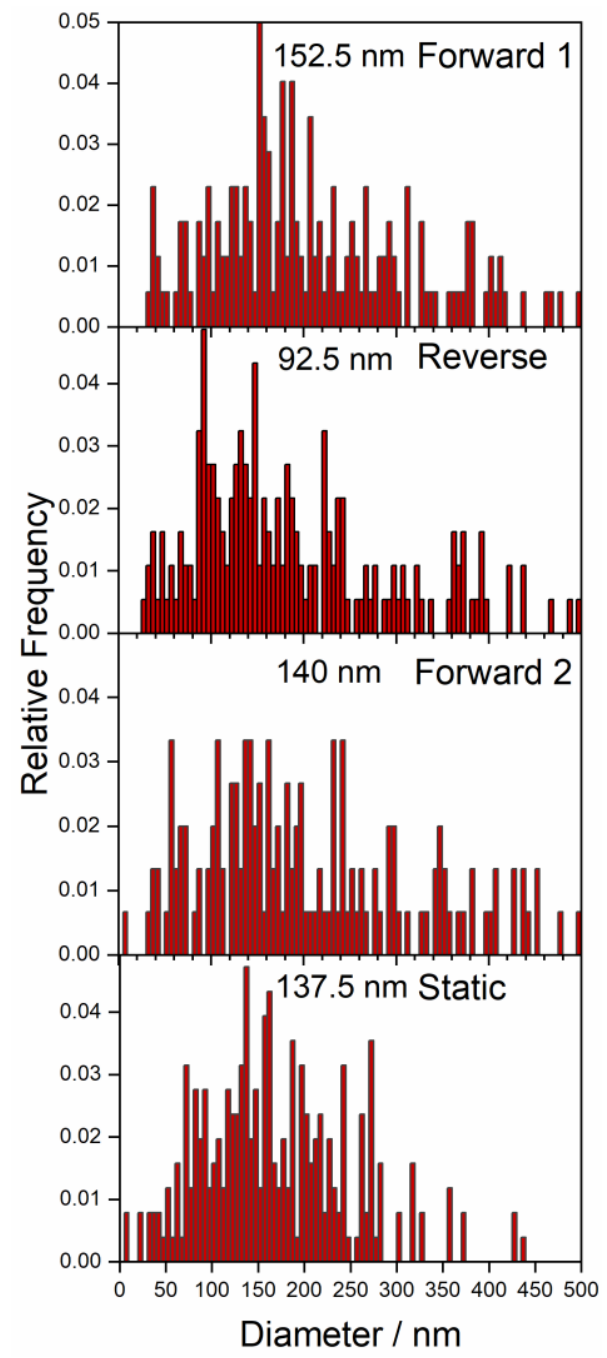

Figure S 10: Size distributions of the NPs with LSPR having hues 0-55 assigned to $\mathrm{SiO}_{2} @$ AuNPs in the mixture and calculated from the 1-D mean square displacement during RMHD-pumping in the forward 1, reverse, and forward 2 directions, as well as in the static suspension (no flow). 


\section{REFERENCES}

(1) Wagner, T.; Lipinski, H.-G.; Wiemann, M. Dark field nanoparticle tracking analysis for size characterization of plasmonic and non-plasmonic particles. J Nanopart Res 2014, 16 (5). DOI: 10.1007/s11051-014-2419-x.

(2) Khan, F. Z.; Fritsch, I. Chip-Scale Electrodeposition and Analysis of Poly(3,4ethylenedioxythiophene) (PEDOT) Films for Enhanced and Sustained Microfluidics Using DC-Redox-Magnetohydrodynamics. J. Electrochem. Soc. 2019, 166 (13), H615-H627. DOI: $10.1149 / 2.0811913$ jes.

(3) Sahore, V.; Fritsch, I. Flat Flow Profiles Achieved with Microfluidics Generated by RedoxMagnetohydrodynamics. Anal. Chem. 2013, 85 (24), 11809-11816. DOI: $10.1021 / \mathrm{ac} 402476 \mathrm{v}$.

(4) Khan, F. Z.; Hutcheson, J. A.; Hunter, C. J.; Powless, A. J.; Benson, D.; Fritsch, I.; Muldoon, T. J. Redox-Magnetohydrodynamically Controlled Fluid Flow with Poly(3,4ethylenedioxythiophene) Coupled to an Epitaxial Light Sheet Confocal Microscope for Image Cytometry Applications. Anal. Chem. 2018, 90 (13), 7862-7870. DOI: 10.1021/acs.analchem.7b05312.

(5) Kestin, J.; Sokolov, M.; Wakeham, W. A. Viscosity of liquid water in the range $-8{ }^{\circ} \mathrm{C}$ to $150{ }^{\circ}$ C. Journal of Physical and Chemical Reference Data 1978, 7 (3), 941-948. DOI: $10.1063 / 1.555581$. 